\title{
ARTICLE
}

\section{Sequencing and de novo assembly of a near complete indica rice genome}

Huilong Du ${ }^{1,2, \star}$, Ying $\mathrm{Yu}^{1, \star}$, Yanfei Ma ${ }^{1, \star}$, Qiang Gao ${ }^{1, \star}$, Yinghao Cao ${ }^{1, \star}$, Zhuo Chen ${ }^{1,2}$, Bin Ma ${ }^{1}, \mathrm{Ming}_{\mathrm{Q}} \mathrm{i}^{1}$, Yan $\mathrm{Li}^{1}$,

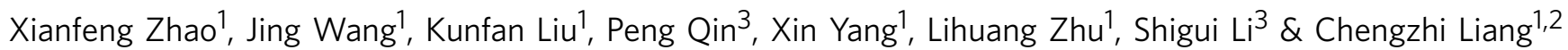

A high-quality reference genome is critical for understanding genome structure, genetic variation and evolution of an organism. Here we report the de novo assembly of an indica rice genome Shuhui498 (R498) through the integration of single-molecule sequencing and mapping data, genetic map and fosmid sequence tags. The $390.3 \mathrm{Mb}$ assembly is estimated to cover more than $99 \%$ of the R498 genome and is more continuous than the current reference genomes of japonica rice Nipponbare (MSU7) and Arabidopsis thaliana (TAIR10). We annotate high-quality protein-coding genes in R498 and identify genetic variations between R498 and Nipponbare and presence/absence variations by comparing them to $17 \mathrm{draft}$ genomes in cultivated rice and its closest wild relatives. Our results demonstrate how to de novo assemble a highly contiguous and near-complete plant genome through an integrative strategy. The R498 genome will serve as a reference for the discovery of genes and structural variations in rice.

\footnotetext{
${ }^{1}$ State Key Laboratory of Plant Genomics, Institute of Genetics and Developmental Biology, Chinese Academy of Sciences, 1 Beichen West Road No. 2, Beijing 100101, China. ${ }^{2}$ College of Life Sciences, University of Chinese Academy of Sciences, Beijing 100049, China. ${ }^{3}$ Rice Research Institute, Sichuan Agricultural University, Chengdu 611130, China. * These authors contributed equally to this work. Correspondence and requests for materials should be addressed to C.L. (email: cliang@genetics.ac.cn) or to S.L. (email: lishigui@sicau.edu.cn).
} 
A ssembly of a high-quality reference genome is the basis for identifying genes and genetic variations, especially structural variations (SVs) which include duplications, insertions, deletions, inversions and translocations. SVs account for more heritable nucleotide variants in a population than singlenucleotide variations and have an important influence on human disease and phenotypic diversity in both animals ${ }^{1,2}$ and plants ${ }^{3,4}$. Next-generation sequencing technologies have been used in the de novo assembly of many plant and animal genomes over the past decade. However, the genomes assembled from short reads are generally draft genomes consisting of up to tens of thousands of contigs, especially in plants, due to high repeat content or low sequencing coverage at complex GC- or AT-rich regions ${ }^{5,6}$. Draft genome assemblies often contain fragmented genes, collapsed or redundant repeats and chimeric contigs that confound gene functional assignment and SV detection. Therefore, improving and validating genome assemblies through the application of up-to-date computational and experimental methods is an important foundation-building task for genomics-driven studies. Single molecule, real-time (SMRT) sequencing on the Pacific Biosciences (PacBio) platform generates long reads of up to $40-60 \mathrm{~kb}$ with randomly distributed errors ${ }^{7}$. This makes SMRT sequencing very suitable for de novo genome assembly under high sequencing depth, and it has been used to assemble complete microbial genomes, high-quality plant draft genomes and significantly improved human and animal reference genomes ${ }^{8-11}$.

However, although the assembled contig length has been significantly improved with the SMRT sequencing data, the problem of fragmentation in the genome assemblies mentioned above still exists. To create chromosome-scale pseudomolecules in genome assembly, in plants, it is common to use a genetic map to order and orient the assembled contigs/scaffolds, but it does not fill gaps and often leaves many misordered, unoriented and unanchored contigs/scaffolds, as well as potentially unfixed assembled errors. A correctly constructed chromosome sequence is critical for gene or quantitative trait locus mapping and genome-wide association studies through linkage disequilibriumbased analysis. Large-insert DNA fragment libraries have played a critical role in sequencing and assembling all high-quality reference genomes in the past, due to their ability to resolve segmentally duplicated regions ${ }^{12}$. There exist several types of large-insert libraries, of which the fosmid library has many advantages over others, such as its shorter construction time, lower cost and better genome coverage resulting from lower cloning bias. However, the high cost involved in sequencing those libraries has largely limited their usage to a small number of model organisms. Furthermore, a recently developed technology, the Irys single-molecule next-generation mapping system from BioNano Genomics, provides physical maps (genome maps) of hundreds of kilobases $(\mathrm{kb})$ to megabases $(\mathrm{Mb})$ in length based on the distribution of nicking sites of endonucleases. Although the genome maps can be used to scaffold assembled sequences or identify SVs for verifying the assembly quality ${ }^{13,14}$, short contigs of up to $100-200 \mathrm{~kb}$ are often not matchable to genome maps due to the shortage of nicking sites, which leads to many large gaps in the scaffolds to be resolved by other methods.

Rice (Oryza sativa, $2 n=2 x=24$ ) is one of the most important crops in the world, providing $19 \%$ of daily caloric intake per capita globally ${ }^{15}$. Traditionally, rice is grouped into two subspecies: indica and japonica, which diverged from their common ancestors $\sim 0.44$ million years ago ${ }^{16}$. The two rice subspecies differ significantly both in morphological traits and at the sequence level, and often show various degrees of intercross hybrid sterility ${ }^{17,18}$. The current rice reference genome was sequenced from the japonica cultivar Nipponbare (Nip) using the clone-based assembly approach ${ }^{19}$. However, indica rice is planted in more areas worldwide than japonica rice, has more genetic diversity and can be further subdivided into indica and aus subpopulations $\mathrm{s}^{20}$. Although several draft genomes of indica rice have been assembled over the past decade ${ }^{21-24}$, their fragmented or incomplete nature has limited their usage in gene or genetic variation discovery. Therefore, a high-quality reference genome for indica cultivars is still required for expanding our knowledge on rice genome function and evolution ${ }^{25}$.

Here, we present a cost-effective method for de novo assembly of high-quality reference genomes by leveraging whole-genome shotgun (WGS) SMRT sequencing, pooled fosmid clone sequencing and genetic map construction, and using BioNano genome maps to verify and help correct the assembled sequences. We assemble a highly contiguous and near-complete indica rice genome of the cultivar Shuhui498 (R498), a restorer line in a three-line hybrid system. We provide high-quality genome annotations for R498 and identify genetic variations by comparing it to the Nip reference genome. We also use both R498 and Nip as a reference to identify presence/absence variations (PAVs) in 17 other deeply sequenced rice genomes. Our method can effectively assemble high-quality complex genomes. This R498 assembly provides an extra resource for gene discovery and for studying genetic variations in rice.

\section{Results}

Genome sequencing and construction of super-contigs. We sequenced R498 genomic DNA to generate $47 \mathrm{~Gb}(\sim 118 \times)$ of WGS SMRT subread sequences from 23 cells using P5 polymerase binding and C3 chemistry and 38 cells using P6 polymerase binding and C4 chemistry, with $\mathrm{N} 50$ of $11 \mathrm{~kb}$ (Supplementary Table 1$)$. The longest $10 \mathrm{~Gb}$ sequences $(\sim 25 \times$ genome coverage) had a length of at least $16 \mathrm{~kb}$. We sequenced an $\mathrm{F}_{3}$ population of 364 individuals produced from a cross between R498 and Nip via genotyping-by-sequencing (GBS) technology ${ }^{26}$. In all, we generated $26.9 \mathrm{~Gb}$ of clean GBS tags of length $2 \times 125 \mathrm{bp}$ with an average of $73 \mathrm{Mb}$ sequences for each $\mathrm{F}_{3}$ individual $(4 \times$ sequencing depth for each tag). We sequenced 564 fosmid pools, each containing $\sim 1,000$ independent clones with insert size of $\sim 40 \mathrm{~kb}$, to produce $6.3 \mathrm{~Gb}$ of GBS tags of length $2 \times 125 \mathrm{bp}(3 \times$ for each tag on average $)$.

We first used the $\mathrm{PBCR}^{27}$ pipeline to correct the raw SMRT sequences, which resulted in $16.2 \mathrm{~Gb}$ of self-corrected sequences. We assembled the corrected sequences on two sets of parameters: default low stringency (LS) and high stringency (HS), which resulted in 436 and $471 \mathrm{Mb}$ of sequences, with contig N50 size of $1.1 \mathrm{Mb}$ and $443 \mathrm{~kb}$, respectively (Supplementary Table 2). We also corrected and assembled the raw SMRT sequences with Falcon and CANU pipelines, resulting in an assembly size of 397 and $405 \mathrm{Mb}$, and contig N50 size of 516 and $900 \mathrm{~kb}$, respectively (Supplementary Table 2). The PBcR LS assembly was used as reference for the construction of genetic map due to its largest N50. The constructed genetic map consisted of 12 linkage groups (LGs), which anchored and ordered 497 contigs, with a total sequence length of $355.9 \mathrm{Mb}$. The fosmid GBS tags were aligned to the PBcR corrected SMRT sequences to retrieve $800 \mathrm{Mb}$ $(\sim 20 \times$ of the pooled fosmid insert size) of the best aligned sequences into each fosmid pool. Each pool of the selected sequences was assembled with Falcon separately and the contigs shorter than $10 \mathrm{~kb}$ were discarded. The resulting fosmid contigs were pooled together to a total size of $39.7 \mathrm{~Gb}$, with N50 size of $42 \mathrm{~kb}$, and max length of $616.8 \mathrm{~kb}$ (Supplementary Table 2). It is worth mentioning that all the fosmid contigs were generated from the WGS SMRT data, but not from fosmid clone sequences, which minimized sequence variations introduced in the cloning process. We aligned all the fosmid contigs to both $\mathrm{PBCR}$ 
assemblies and found that $<5 \%$ of the aligned fosmid contigs were chimeric (Supplementary Fig. 1a) and the average alignment identity was $>99 \%$, suggesting that the fosmid contigs were of high quality for connecting the WGS contigs.

As fully described in the Methods section, the WGS contigs on the genetic map were connected or extended into super-contigs iteratively by selecting the best paths on the overlap graphs that were constructed using WGS contigs as nodes and fosmid contigs as edges (Fig. 1). We minimized connection errors by using each WGS contig only once and carefully prioritize the node pairs to be connected at any stage (Supplementary Note 1). Wholegenome assembly often produces misassembled chimeric contigs. Those contigs were captured during the merging process if they were aligned with an overhang of $>1 \mathrm{~kb}$ to its adjacent contigs with high sequence identity $(>98 \%)$ and the overlap was also supported by fosmid contigs. The identified chimeric contigs were

a

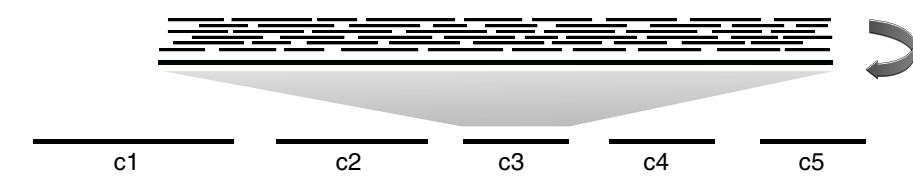

b

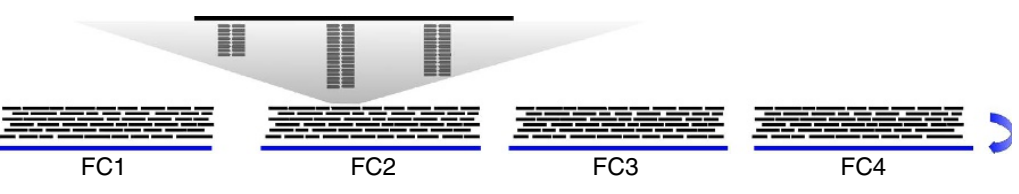

C

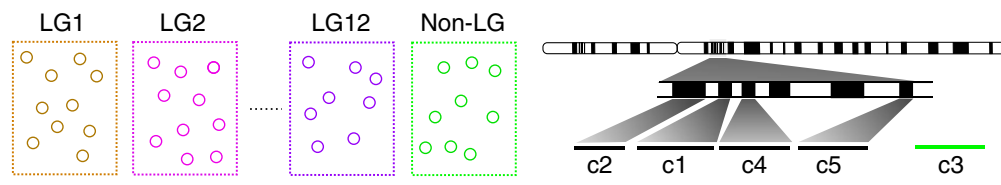

d
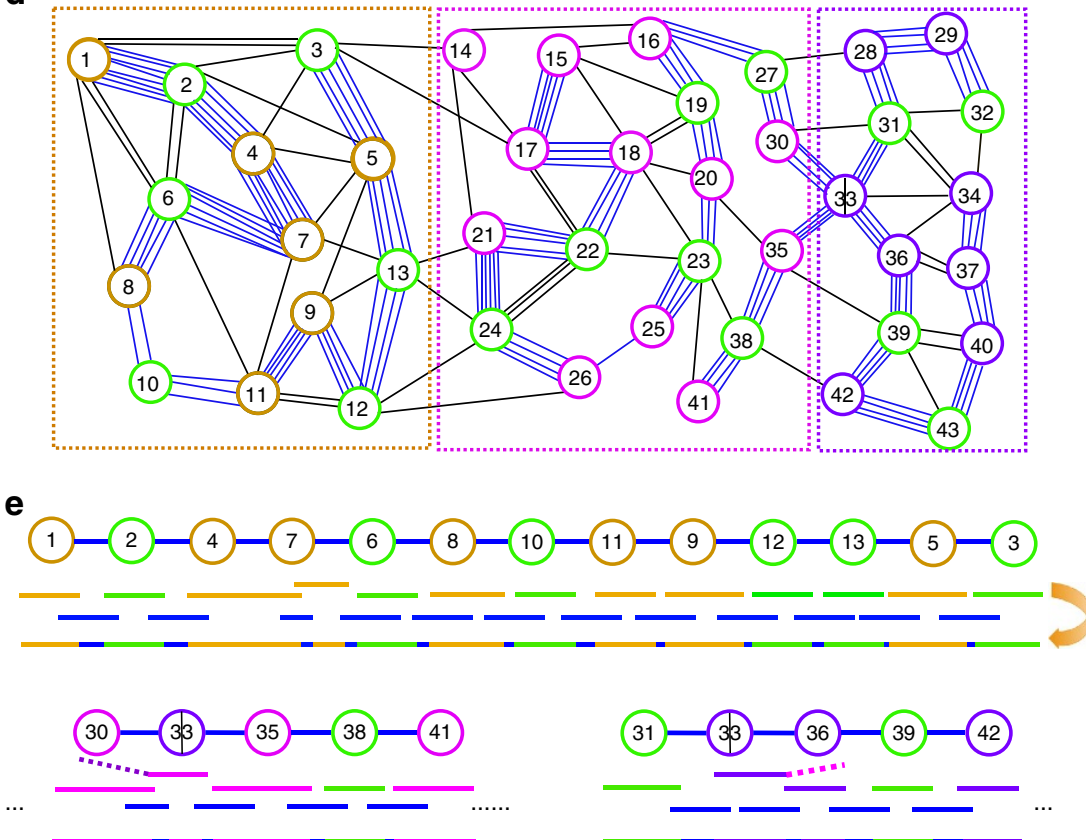

Figure 1 | Schematic depiction of the construction of super-contigs. (a) Whole-genome assembly from corrected SMRT sequences (thin black lines) generates a set of WGS contigs (c1-c5). (b) Sequence tags (stacked short lines) from each fosmid pool are used to retrieve corrected PacBio sequences (black lines), which are then assembled into fosmid contigs (for example, FC1-FC5). (c) Many of the WGS contigs are grouped together and anchored onto chromosomes based on their linkage relationship (LG1-LG12). For example, c1, c2, c4 and c5 are anchored onto a chromosome, but c3 is not (in the non-LG group). Note that c2 and c4 are close to c1 physically, but c5 is not close to them. (d) A simplified overlap graph is constructed using anchored WGS contigs in LG1, LG2 and LG12 and unanchored WGS contigs in the non-LG group as nodes and fosmid contigs as edges (blue lines). A sequence overlap between a WGS contig and a fosmid contig must be at least $5 \mathrm{~kb}$ to form a connection. Two WGS contigs can be connected by multiple fosmid contigs. (e) Three most reliable paths from (d), including two partial ones, are selected as described in the Methods section to build super-contigs. The blue lines represent fosmid contigs. The other coloured lines represent WGS contigs. The WGS contigs (including unanchored ones in green) on each path are connected to the best aligned fosmid contigs to form super-contigs. The overlap between two WGS contigs (for example, nodes 4 and 7 ) causes negative edge length as described in the Methods section. In such cases, the fosmid sequences were used for the overlapping regions to form the super-contig. In all other cases, the WGS contig sequences were used for the overlapping regions. Node 33 represents a chimeric WGS contig, which is split into two parts to be used separately in two super-contigs. Dashed lines represent alignment overhangs. 
split into multiple contigs at the detected joining positions, and the cleaved sequences longer than $30 \mathrm{~kb}$ were reused elsewhere as new WGS contigs (Fig. 1e).

For building super-contigs, we found that although the LS contigs had higher N50 size, the HS contigs were better due to their higher accuracy (Supplementary Note 1). The resulting genome based on the HS contigs consisted of 17 super-contigs (a total of $390.6 \mathrm{Mb}$ ), with seven chromosomes assembled into single super-contigs and the remaining five each assembled into two super-contigs. We detected a long stretch of the telomeric sequence $5^{\prime}$-TTTAGGG- $3^{\prime}$ at all chromosome ends, suggesting the completion of every chromosome arm at the end.

The 17 super-contigs incorporated 1,693 PBcR HS contigs and 1,676 fosmid connections, including 378 connections supported by both sequence overlaps between WGS contigs and fosmid contigs (Fig. 1e). During the connection process, 36 and 13 chimeric contigs were identified and split in the PBcR LS and HS assemblies, respectively. Of the 497 anchored LS contigs on the genetic map, we found that 127 contigs (a total of $45.3 \mathrm{Mb}$ ) were repositioned relative to other contigs and 272 contigs (a total of $73.7 \mathrm{Mb}$ ) were reoriented on the super-contigs.

Correction of errors in the super-contigs. We obtained a total length of $99 \mathrm{~Gb}(250 \times)$ of BioNano single-molecule maps ( $>100 \mathrm{~kb}$ ) with $\mathrm{N} 50$ size of $202 \mathrm{~kb}$, which were de novo assembled to 453 genome maps with a total size of $406 \mathrm{Mb}$ and N50 of $1.22 \mathrm{Mb}$ (Supplementary Data 1). The relative short length of the genome maps was caused by the existence of common breaking points in the raw single-molecule maps ${ }^{14}$. We also conducted a hybrid assembly by combining the PBcR LS contigs and the BioNano maps, which generated sequence scaffolds that were represented by 225 genome maps with a total size of $408 \mathrm{Mb}$ and $\mathrm{N} 50$ size of $2.48 \mathrm{Mb}$. Since short contigs (with $<8$ nicking sites) were not aligned to genome maps, the scaffolds contained many gaps of up to a few hundred kb. Notably, it is possible to build super-contigs under the guidance of hybrid genome maps using the method described above, but the genetic map is still required to anchor the super-contigs onto chromosomes. Therefore, in this study we only used the genome maps to assess the quality of the super-contigs constructed under the guidance of genetic map and to help correct potential assembly errors in the super-contigs.

For convenience we added $10 \mathrm{~kb}$ of ' $\mathrm{N}$ 's between each pair of contigs on the same chromosome to connect them into pseudomolecules and aligned the de novo assembled genome maps to them. The genome maps (446 of 453) covered $96.62 \%$ of the 12 pseudomolecules with 226 uncovered gaps $>0 \mathrm{bp}$ (a total of $13.2 \mathrm{Mb}$ with $\mathrm{N} 50$ size of $125 \mathrm{~kb}$ and $\max 495 \mathrm{~kb}$; Supplementary Fig. 2). For quality check, we first examined the most error-prone regions in the genome: centromeres and subtelomeres. The successfully assembled seven centromeres in $\mathrm{R} 498$ were confirmed to be correct but none of the five gaps in the centromeric regions were covered completely by a single map (Supplementary Fig. 3). Of the 24 assembled chromosome ends, 21 subtelomeres were also confirmed correct (Supplementary Fig. 4). There were potentially missing subtelomeric repeats in the distal end of chromosome 3,10 and 11. The nucleolus organizer regions were found to be located at the start of two pseudomolecules 9 and 10, which contained six and nine assembled copies of 17S-5.8S-25S ribosomal RNA genes (rDNAs), respectively (Supplementary Fig. 4). One genome map was aligned to chromosome 9 , which contained 22 copies of rDNAs (Supplementary Fig. 4). We found 25 maps aligned to the start of chromosome 10, of which 24 (a total of $17.1 \mathrm{Mb}$ ) appeared to be rDNA-only maps (Supplementary Fig. 4), and the only map that was extended to non-rDNA region contained a tandem array of
40 rDNAs. We identified telomere repeats immediately adjacent to rDNAs in many SMRT reads, confirming the chromosomal end location of rDNAs. Due to their sequence complexity, we did not try to fix the missing sequences in these two rDNA regions.

Next, we identified SVs in the pseudomolecules as potential assembly or connection errors compared with the genome maps (Supplementary Fig. 5 and Supplementary Table 13). Excluding the two rDNA regions, we found 44 insertions and deletions (indels) longer than $10 \mathrm{~kb}$ (max length $175 \mathrm{~kb}$ ), including 17 insertions (a total of $350 \mathrm{~kb}$ ) and 17 deletions (a total of $1.6 \mathrm{Mb}$ ), and 63 indels of $1-10 \mathrm{~kb}$ (a total of $410 \mathrm{~kb}$ ) in the pseudomolecules. The genome maps fully covered 1,621 (96.72\%) connecting regions, of which 74 were present in 54 indels (34 indels $>10 \mathrm{~kb}$ ), indicating a max error rate of $4.57 \%$ for misconnections (note that not all connections were wrong in the indels involving multiple connections). As a comparison, the other 53 indels $(10$ of them $>10 \mathrm{~kb}$ ) were present in the original $\mathrm{PBcR}$ HS contigs. We found that all the potential misconnections were local errors mostly with missing repetitive sequences, that is, none of the genome maps was aligned to two non-adjacent genome locations.

Of the indels $>10 \mathrm{~kb}, 30$ were corrected by choosing an alternative route of the correct length on the overlapping graph with matching nicking sites to replace the erroneously incorporated contigs (Supplementary Fig. 5). The 14 uncorrected indels (a total size of $1 \mathrm{Mb}$ ), including 11 in connecting regions, were either long tandem repeats such as one with $>60 \mathrm{~kb}$ repeat unit on chromosome 5 or complex repeats such as those in the subtelomere regions (Supplementary Fig. 6). To fix the potential indel errors of $1-10 \mathrm{~kb}$, we aligned the corrected SMRT reads to each region and conducted local assembly with the aligned sequences. We found that 46 of the regions were reassembled correctly by comparing the newly assembled contigs to the genome maps, so we used the new contigs to replace the incorrect sequences. The remaining 17 indels, including 3 in connecting regions, were not changed because the local reassembly still generated the same sequences. After correction of the indels, the total number of connecting regions was reduced to 1,652 , which connected 1,669 HS contigs in the final pseudomolecules.

Finally, the assembled genome sequences were corrected by Quiver 8 , which fixed minor sequence errors up to $1-2 \mathrm{~kb}$. We then aligned $38.7 \mathrm{~Gb}(\sim 98 \times)$ of Illumina short reads to the pseudomolecules (genome coverage of $99.88 \%$ ) to identify and fix 40,784 homozygous indels of $1-2 \mathrm{bp}$, which were resulted from the non-random indel errors in homopolymer runs in SMRT sequences. The resulting genome sequences were $390,322,188 \mathrm{bp}$ (including $50 \mathrm{~kb}$ of ' $\mathrm{N}$ 's; GC content, 43.57\%). We realigned the genome maps to the pseudomolecules (Supplementary Data 2) to recompute the coordinates of the error-prone regions (Supplementary Table 3).

Assessment of the sequence quality. We estimated the base error rate in the genome to be $<0.0017 \%$ with both Illumina DNA short reads and RNA sequencing (RNA-seq) data (Supplementary Table 4). This error rate was well below one error per 10,000 bases, a quality standard used for human genome $e^{28}$ and other high-quality genomes such as Nipponbare ${ }^{19}$. The heterozygous single-nucleotide polymorphisms (SNPs) and indels represented genome heterozygosity of $0.0038 \%$ in our sequenced R498 samples, suggesting that the R498 genome was highly homozygous.

The fosmid contigs contributed a total of $11.9 \mathrm{Mb}(3.05 \%)$ sequences in the final pseudomolecules, with N50 size of $13.9 \mathrm{~kb}$ and a max of $81.9 \mathrm{~kb}$ for a single connecting region. Using Illumina short reads, the error rate in the connecting regions 
(with $98.21 \%$ being covered) were estimated to be $0.0071 \%$, which was slightly larger than the whole-genome average, most likely due to the repetitive nature of these regions. Using the pseudomolecules as reference, we found that the average base accuracy of all the fosmid contigs was $99.33 \%$ and comparable to that of the WGS contigs (Supplementary Fig. 1b). We also found that the chimeric fosmid contigs were randomly distributed throughout the genome (Supplementary Fig. 1c). These results further confirmed that the potential errors introduced by the fosmid contigs were minimal.

The $\mathrm{PBCR}$ HS assembly was found to be highly redundant compared with the Falcon or CANU assemblies, but had the highest sensitivity $(99.75 \%)$ and the lowest error rate $(0.57 \%)$ (Supplementary Note 2 and Supplementary Table 5). Of the 471.1 Mb sequences in the PBcR HS assembly, only $3 \mathrm{Mb}$ of centromeric sequences and $1 \mathrm{Mb}$ of unclassified sequences were not mapped to the pseudomolecules (Supplementary Table 2). Almost all the unclassified contigs were found to be assembled from partially corrected sequences during $\mathrm{PBcR}$ self-correction, as suggested by their low coverage of Illumina short reads (Supplementary Fig. 7). Based on these results and the comparison results to genome maps, we estimated that $<1 \%$ of the R498 genome sequences were missing in the pseudomolecules (Supplementary Note 3).

Assembly of two organelle DNAs. The R498 chloroplast DNA (cpDNA) and mitochondrial DNA (mtDNA) were initially connected into two misassembled contigs of 593 and $618 \mathrm{~kb}$, respectively. We selected the corrected PacBio reads that were aligned to these two contigs with a minimum aligned length of $3 \mathrm{~kb}$ and a minimum alignment identity of $98 \%$ for reassembly. The cpDNA was directly assembled into a circular contig containing a non-redundant sequence of $134.5 \mathrm{~kb}$. The mtDNA was assembled into a circular DNA after several rounds of extension and local reassembly, and connection with fosmid contigs, as described in the Methods section. The resulting non-redundant mtDNA was $527.1 \mathrm{~kb}$, which is $36.5 \mathrm{~kb}$ longer than that of Nip (Table 1). We found that the Nip mtDNA was potentially misassembled, most likely due to the complex internal repeat structures that included three sets of large repeats longer than $20 \mathrm{~kb}$ (Supplementary Fig. 8). Comparison with genome maps showed that the cpDNA was fully covered by genome maps, but unfortunately the mtDNA was only partially covered by one map of $117 \mathrm{~kb}$.

Gene and repeat annotation. We predicted 38,714 high-confidence protein-coding genes in R498 through an evidence-based gene prediction pipeline $\mathrm{e}^{29}$ by combining known proteins, experimentally cloned cDNAs/expressed-sequence tags (ESTs) and assembled transcripts from rice RNA-seq data (see Methods for detailed description). Using RNA-seq data from five tissues types of R498 (Supplementary Table 1), we found that 32,393 (83.67\%) of these genes were expressed in at least one of the samples. For unbiased gene comparison between R498 and Nip, we also used the same method to annotate 36,775 protein-coding genes in Nip (Supplementary Table 6). We assigned Pfam motifs or functions to $84.82 \%$ and $88.06 \%$ of R498 and Nip genes, respectively (Supplementary Table 7). The mutual comparison among our predicted Nip gene set, MSU7 set and RAP set (http://rapdb.dna.affrc.go.jp/) showed that these three gene sets are largely similar to each other with extra unshared genes in each set (Supplementary Fig. 9). Since all our predicted genes have protein homology, cDNA/EST or RNA-seq data as evidence, and each exon were predicted based on sequence alignment, these extra genes in our annotation are very likely true genes that are missing in the other gene sets.

We annotated the repetitive sequences in both R498 and Nip by combining de novo prediction and homology-based search at both the DNA and protein levels. The repetitive sequences account for $42.05 \%$ and $40.43 \%$ of the R498 and Nip genomes, respectively (Supplementary Table 8). Our annotation of $98.9 \mathrm{Mb}$ long terminal repeat (LTR)-retrotransposons in Nip corresponds well to the previous estimation of at least $97 \mathrm{Mb}$ in the Nip genome $^{30}$. We annotated more repeated sequences in Nip than MSU7 annotation (34.23\%), probably owing to the use of multiple repeat libraries in our method.

Whole-genome comparison to Nip reference genome. The R498 assembly was $17 \mathrm{Mb}$ longer and contained fewer gaps than the current Nip reference genome MSU7 (http://rice.plantbiology.msu.edu, release 7, $373 \mathrm{Mb}$ ) (Table 1). Except chromosome 12, all other 11 chromosomes of R498 are longer than Nip.

Table 1 | Comparison of basic sequence statistics of R498 and Nipponbare MSU7.

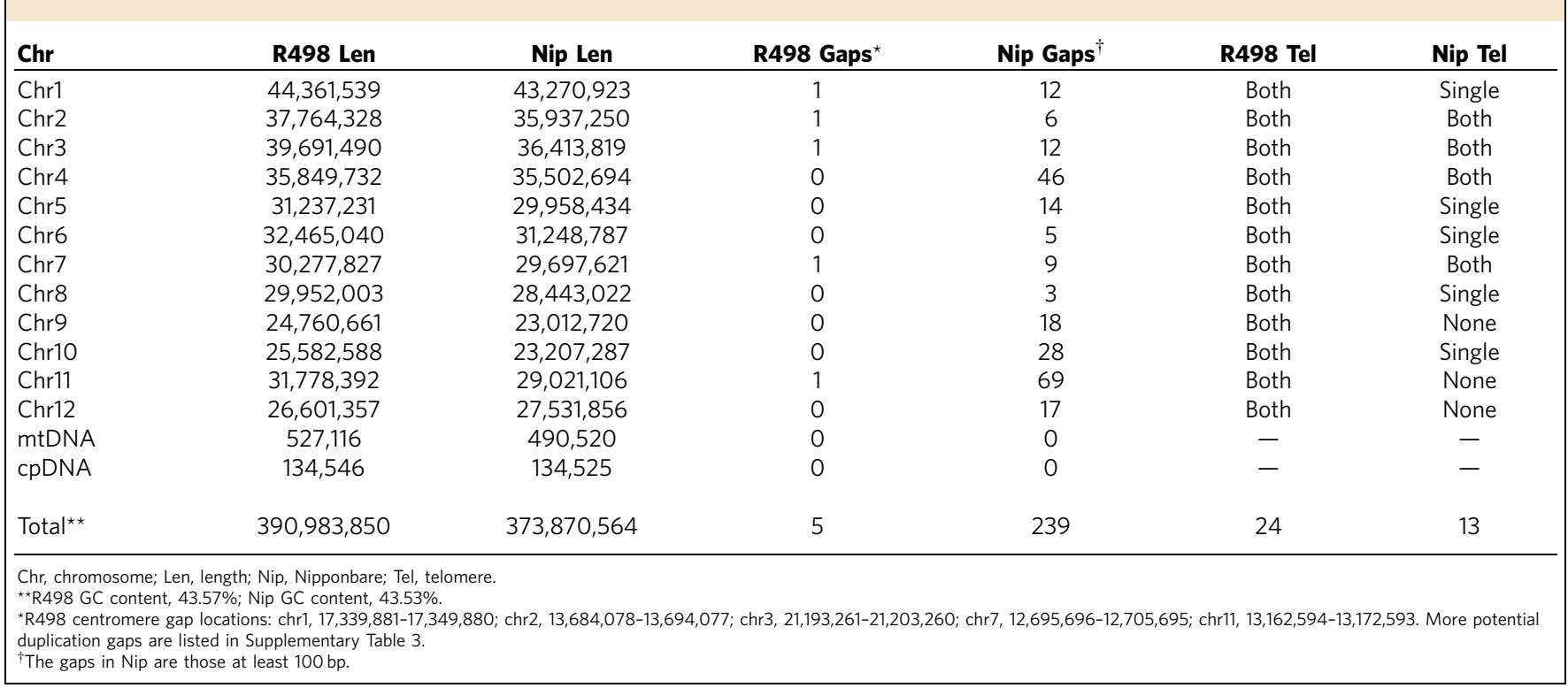


Comparing to Nip, R498 assembly has more completed centromere regions (7 versus 2) (Supplementary Table 9) with considerable divergence between them (Supplementary Fig. 10), more telomeres (24 versus 13; Table 1 ), more assembled rDNAs at the beginning of chromosomes 9 and 10 (Supplementary Table 10) and fewer cpDNAs and mtDNAs (Supplementary Data 3). We aligned the R498 genome sequences to Nip and found their high chromosome-level similarity, with $313.5 \mathrm{Mb}(80.31 \%)$ of R498 sequences aligned to $307.8 \mathrm{Mb}(82.73 \%)$ of Nip sequences in syntenic blocks, and also significant sequence divergence with more than two and half million SNPs (Fig. 2 and Table 2). We identified a large number of SVs between the two genomes. The most remarkable difference between the two genomes was an inversion across the centromere from 12.7 to $18.5 \mathrm{Mb}$ on chromosome 6 (Fig. 2, Supplementary Table 11 and Supplementary Fig. 11a). Both boundaries of this inversion in R498 were confirmed by genome maps (Supplementary Fig. 11b). The alignments of Nip PacBio sequences (http://schatzlab.cshl.edu/data/ ectools/) to Nip genome suggested that the Nip sequences were probably also correct. We also found three smaller inversions of a few hundred kilobase whose boundaries were all validated with genome maps in R498 (Supplementary Fig. 11c,d). We found 8,402 presence variations (PVs) in $\mathrm{R} 498$ (a total of $66 \mathrm{Mb}$, max $484.9 \mathrm{~kb}$ ) and 7,119 PVs in Nip (a total of $39.3 \mathrm{Mb}$, $\max 228.5 \mathrm{~kb}$ ) at least $500 \mathrm{bp}$, which include 170 and $92 \mathrm{PVs}$ that are at least
$50 \mathrm{~kb}$ in R498 and Nip, respectively (Supplementary Data 4). As expected, we identified much more large PAVs than those reported previously ${ }^{23,24}$ owing to the completeness of the genome. A large PV in Nip was found on chromosome 12 from $20,108,154$ to $20,783,761 \mathrm{bp}$. Further inspection revealed a potential misassembly in the Nip genome from 20,460 to $20,705 \mathrm{~kb}$, which contained a piece of $245 \mathrm{~kb}$ mitochondria-like DNA, which was aligned to Nip mtDNA with $>99.1 \%$ identity in interleaved blocks (Supplementary Data 3).

Comparing our predicted two gene sets showed substantial variations in genic regions between the two genomes (Fig. 3a). There are only 10,244 (26.46\%) genes in R498 that share identical proteins with 10,289 (27.98\%) Nip genes (not a 1:1 relationship). At minimum sequence coverage and identity of both $50 \%$, there are 29,686 (78.68\%) genes in R498 and 30,290 (82.36\%) genes in Nip having a homologue in its counterpart, and at both $90 \%$, there are only $24,098(62.24 \%)$ and 24,203 (65.81\%) homologous genes between the two genomes. We searched the existence of 1,017 known rice gene alleles, including some on the same locus, by aligning their protein sequences to R498 and Nip, and found the majority of them to be different at the protein level between the two genomes and that more than 300 of them are identical to R498 (Supplementary Data 5). There are 66 gene alleles identical to R498 that are different in Nip, including GW5 (ref. 31), a gene associated with rice grain width and weight, PSTOL1 (ref. 32)

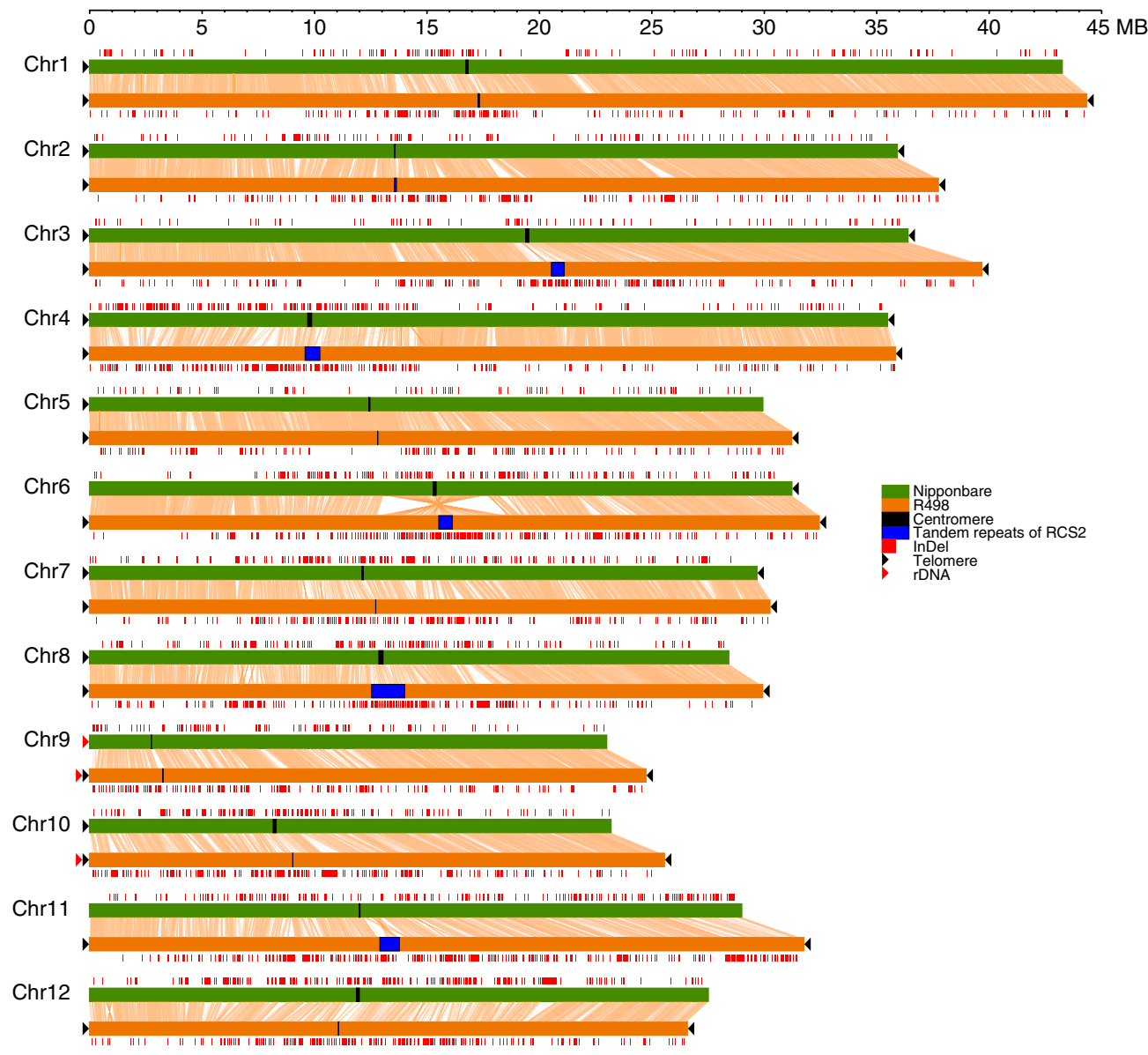

Figure 2 | Whole-genome comparison of R498 and Nip. The aligned regions are represented by the crossing lines between each pair of pseudomolecules. A large inversion is observable on chromosome 6 . Red rectangles above or below the pseudomolecules represent PVs ( $\geq 500$ bp) relative to each other. Black rectangles indicate the position of centromere-surrounding sequences defined in Nip reference genome (http://rice.plantbiology.msu.edu/ annotation_pseudo_centromeres.shtml), and blue rectangles represent the regions containing centromere-specific tandem repeats of RCS2 in R498. Left or right black arrows at the end of each pseudomolecule indicate the presence of telomere repeats. Red arrows indicate the locations of rDNAs at the start of chromosomes 9 and 10 . 


\section{Table 2 | Comparison of genome features between R498 and Nipponbare MSU7.}

\begin{tabular}{|c|c|c|}
\hline Feature type & In R498 & In Nip \\
\hline \multicolumn{3}{|l|}{ Gene } \\
\hline Number & 38,714 & 36,775 \\
\hline \multicolumn{3}{|l|}{ Repeat content } \\
\hline$\%$ & 42.05 & 40.43 \\
\hline \multicolumn{3}{|l|}{ SNP } \\
\hline Number & $2,548,071$ & $2,548,071$ \\
\hline \multicolumn{3}{|c|}{ Insertion of 1-99 bp } \\
\hline Number & 226,771 & 239,390 \\
\hline Base & $1,034,898$ & $1,077,080$ \\
\hline \multicolumn{3}{|c|}{ PV of $100-499 \mathrm{bp}$} \\
\hline Number & 3,432 & 3,411 \\
\hline Base & 887,602 & 856,902 \\
\hline \multicolumn{3}{|c|}{ PV of 500-999 bp } \\
\hline Number & 1,301 & 1,328 \\
\hline Base & 938,805 & 962,372 \\
\hline \multicolumn{3}{|c|}{$P V$ of $\geq 1-10 \mathrm{~kb}$} \\
\hline Number & 5,407 & 4,973 \\
\hline Base & $19,316,383$ & $16,710,125$ \\
\hline \multicolumn{3}{|c|}{$P V$ of $\geq 10-50 \mathrm{~kb}$} \\
\hline Number & 1,524 & 726 \\
\hline Base & $28,857,940$ & $13,663,983$ \\
\hline \multicolumn{3}{|l|}{$P V$ of $\geq 50 \mathrm{~kb}$} \\
\hline Number & 170 & 92 \\
\hline Base & $16,851,530$ & $7,960,212$ \\
\hline \multicolumn{3}{|c|}{$P V$ of $\geq 500 \mathrm{bp}$} \\
\hline Number & 8,402 & 7,119 \\
\hline Base & $65,964,658$ & $39,296,692$ \\
\hline
\end{tabular}

$A V$, absence variation; $P V$, presence variation; SNP, single-nucleotide polymorphism An insertion in R498 is equivalent to a deletion in Nip, and vice versa. A PV in R498 is equivalent to an $\mathrm{AV}$ in Nip, and vice versa.

(Fig. 3b), a gene conferring tolerance of phosphorus deficiency, and several blast resistance genes such as Piz-t (Fig. 3c), Pi-km1, Pi5-1 and Pi5-2. We found more genes in the centromere regions (194 versus 182) and subtelomere regions (437 versus 400) of R498 than Nip (Supplementary Table 9). Moreover, we found that 990 and 966 of our annotated genes were fully contained in the PVs in R498 and Nip, and showed low sequence similarity to the other genome, of which 541 (54.65\%) and 381 (39.44\%) were annotated with gene ontology (GO) terms, respectively. The enriched R498-specific GO categories include protein phosphorylation, telomere maintenance, transferase activity for transferring phosphorus-containing groups and protein kinase activity (Supplementary Table 12). Of these R498 genes, 605 (61.11\%) were found to be expressed in at least one of the eight samples in the R498 RNA-seq data (Supplementary Table 1), and $842(85.05 \%)$ are homologous to other R498 genes.

Although the two genomes contain a similar amount of repetitive sequences, they displayed a significant difference in repeat content, for example, Nip had $17.66 \%$ and $4.80 \%$ LTR/ Gypsy and LTR/Copia elements, respectively, while R498 had $20.55 \%$ and $3.86 \%$, respectively (Supplementary Table 8 ). We found almost six times more solo-LTRs $(52,747$ versus 7,823$)$ and slightly more full-length LTR elements $(7,274$ versus 6,686$)$ in R498 than Nip. These results confirmed that rice genomes in two subspecies had experienced independent amplification or loss of LTR retrotransposons after their divergence ${ }^{16}$ (Fig. 3b,c).

Whole-genome comparison with other rice genomes. We compared 17 deeply sequenced rice draft genomes (including $8 \mathrm{O}$. sativa and 9 O. rufipogon) with both R498 and Nip reference genomes to assess the sequence quality of the draft genomes and the diversity of PAVs in rice population (Supplementary Data 6). We found that the R498 sequences were highly similar to the MH63 and ZS97 genomes (Supplementary Fig. 12), with the majority of the known genes in R498 being identical either to MH63 or to ZS97 (Supplementary Data 5), except that the latter two genomes were incomplete ${ }^{24}$. In 93-11, many of the unanchored sequences were aligned to R498 pseudomolecules (Supplementary Fig. 13). We examined the completeness of the 93-11 sequence using the known genes. We found 50 genes, including $W R K Y 1, R P L 12-2, R C g 2$ and $R P K$, being partial in 93-11 but with complete sequences in R498, MH63 and ZS97 (Supplementary Data 5), suggesting the incomplete gene assembly in 93-11.

For PAV identification, we focused on only the PVs on the reference genomes, that is, the AVs on the draft genomes, due to the small contig size of most of the draft genomes. We identified from 1,102 to $3,688 \mathrm{PVs}$ on R498 and from 204 to 2,980 PVs on Nip of $\geq 500 \mathrm{bp}$ relative to the 17 genomes (Supplementary Data 6). As expected, we found fewer PVs between the varieties within subspecies than between subspecies. For example, relative to Koshihikari, a japonica variety, only 204 PVs were identified on Nip, but 3,289 PVs were found on R498. Many of these PVs were different from those found between R498 and Nip, and many of them were largely shared by multiple genomes and present in both $O$. sativa and O. rufipogon (Fig. $3 \mathrm{~d}$ and Supplementary Data 7 and 8). On the other hand, the majority (83.48\%) of the AVs on each draft genome relative to R498 and Nip were on different positions (Supplementary Data 6). These results indicate that different PAVs are also widely present between rice genomes due to genome-specific gain or loss of DNA fragments, similar to the results previously reported in maize ${ }^{4}$, even though rice has much less genomic diversity than maize at population level.

\section{Discussion}

We here present a highly contiguous and near-complete genome assembly for indica rice cultivar R498 through a cost-effective integrated strategy. This assembly contains five centromere gaps and up to 14 potential duplication gaps (Supplementary Table 3), and thus is more continuous than the current reference sequences of Nip, Arabidopsis thaliana (https://www.arabidopsis.org, TAIR10 with 96 gaps) and Brachypodium distachyon (http://www.brachypodium.org/, v3.1 with 24 gaps) that were obtained by clone-based assembly methods. The high contiguity of the assembly was achieved through combination of linkage map-assisted contig grouping/ordering and fosmid clone-assisted contig connections. The connection process performed the following functions: (1) fixed misordered or unoriented WGS contigs anchored on genetic map, (2) placed unanchored WGS contigs onto the right positions on chromosomes, (3) identified and split chimeric contigs generated in the whole-genome assembly, (4) connected immediately adjacent contigs into super-contigs using fosmid contigs and (5) removed redundancy present in WGS contigs. We used BioNano genome maps to validate the high accuracy of the method and help correct many of the assembly or connection errors. The key to the success of this method is that many globally unassembled repetitive regions (especially segmental duplications) due to conflicts on assembling 
a

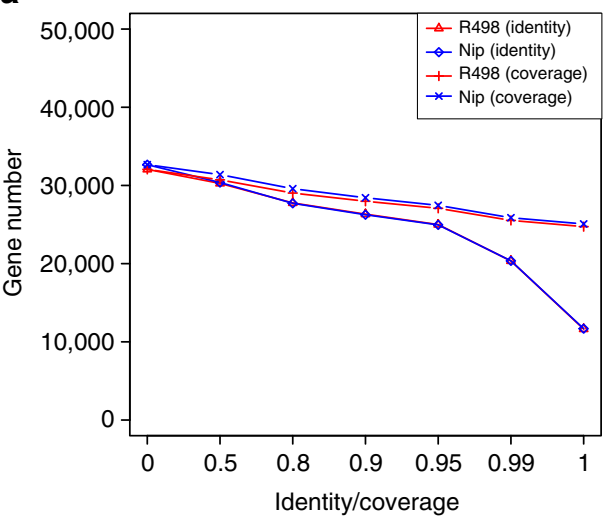

d

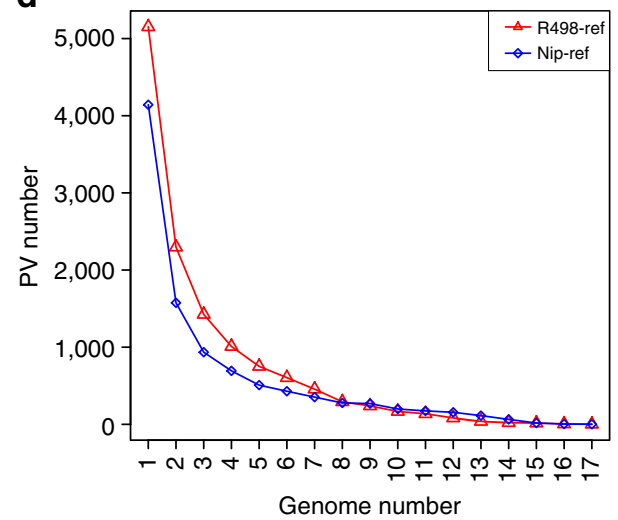

b

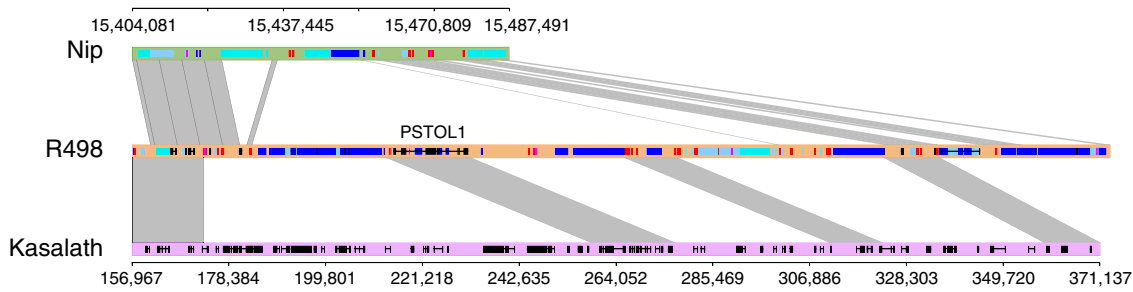

C

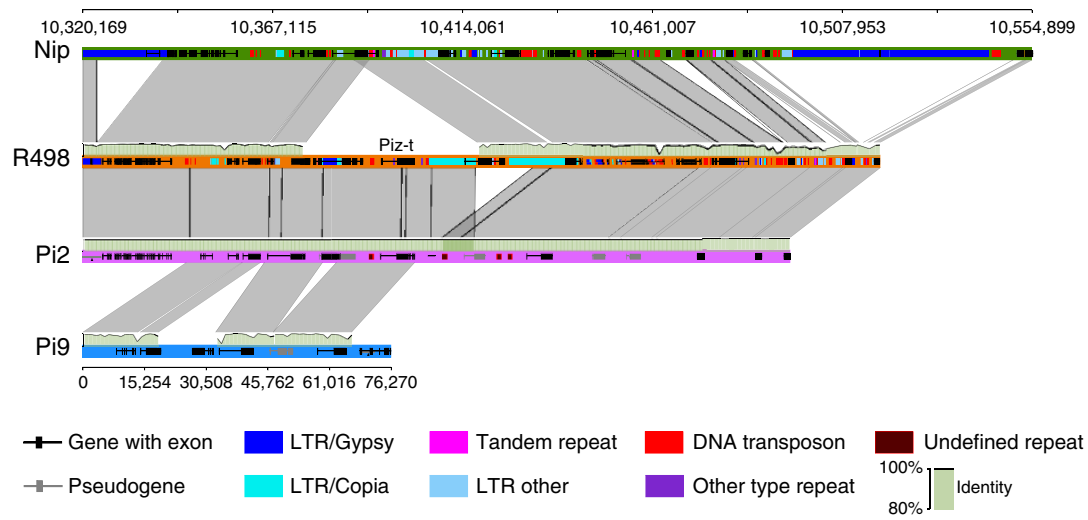

Figure 3 | Comparison of genes and PAVs among rice genomes. (a) Line plot showing the number of homologous genes between R498 and Nip. The proteins coded by genes in R498 and Nip were BLASTed against each other, and the mutual sequence coverage and alignment identity of the best-matched protein pairs was computed. The total number of genes compared was: R498, 38,714; Nip 36,775. The $x$ axis indicates the percentage of either coverage or identity as the threshold. The $y$ axis indicates the number of the genes in R498 or Nip that are aligned to best-matched homologous genes in the other genome with coverage or identity above the threshold. (b) Synteny view of the PSTOL1 gene region between R498 (Chr12: 14,818,743-15,035,152 bp), Nip and Kasalath (GenBank accession no. AB458444.1). Different amplification or loss of LTR retrotransposons between R498 and Nip is evident. (c) Synteny view of rice blast resistance gene locus for Piz-t in R498 (Chr6: 10,079,870-10,277,132 bp), Pi2 from indica cultivar C101A51 and Pi9 from O. minuta (GenBank accession nos DQ352453 and DQ285630) to Nip on chromosome 6. This region is composed of multiple NBS-LRR domain genes, transposons and pseudogenes. Several PAVs of 13-65 kb were shown, which contain different type of LTR transposons. (d) Statistics of the PVs on R498 and Nip that are shared by one or more of the 17 draft genomes. The names and data sources of the 17 draft genomes were listed in Supplementary Data 6. Two PVs on a reference genome relative to two draft genomes were defined as shared if at least $75 \%$ of their sequences overlap. The $y$ axis indicates the number of PVs that are shared under each genome number on $x$ axis.

graphs could be resolved in a local context, either with the help of neighbouring information of contigs on the linkage map, or through the reduced genome complexity in a small portion of the genome (that is, fosmid clones), or by the combination of both.

It is well known that plants have a greater number of complex repetitive sequence structures than animal genomes. The size of plant genomes varies by four orders of magnitude, up to $152 \mathrm{~Gb}^{33}$. To resolve the repeat regions presents a major challenge for plant genome assembly. Although the rice genome is small, our wholegenome assembly generated very short contigs (maximum N50 of $1.1 \mathrm{Mb}$ from PBcR) compared with the assemblies of many other species ${ }^{11,27}$, suggesting that rice is a highly complex genome, possibly due to its well-preserved ancient whole-genome duplications ${ }^{34}$ or recently amplified retrotransposons ${ }^{16}$. Therefore, the strategies used here should be generally adaptable to the assembly of larger plant or animal genomes. Furthermore, our reported work here can also be viewed as proof-of-concept on how to create high-quality and near-complete platinum genomes, as discussed by Chaisson et al. ${ }^{12}$, by leveraging easily accessible technologies. Specifically, the method can be easily extended by replacing genetic map with $\mathrm{Hi}-\mathrm{C}$ map $^{35}$ or replacing pooled fosmid clones with pools of 10 DNA fragments of $>50 \mathrm{~kb}$ that are 
generated by recently developed Chromium Genome system from $10 \times$ Genomics $^{36}$.

This R498 assembly provides an extra resource for gene discovery and for studying genetic variations and genome evolution in rice. We found several genes being present in R498 but missing in Nip and that the majority of the genes have sequence variations between the two genomes. Compared with Nip, the R498 assembly showed superior quality in many repetitive regions. We found one more nucleolus organizer region on chromosomes 10 , more genes in the centromere and subtelomere regions and more LTR elements in R498 than in Nip. We assembled a complete R498 mitochondrial genome that was longer and more accurate than that of Nip. We also found that the amount of the organelle DNAs integrated into the rice nuclear genome might be less than previously observed in Nip. The nearcomplete chromosome-scale R498 genome serves as an ideal reference for linkage-based mutant gene identifications ${ }^{37}$ and genome-wide association studies in indica rice subpopulations.

\section{Methods}

Plant materials and DNA/RNA extraction. O. sativa ssp. indica $(2 n=2 x=24)$ is one of the two major rice subspecies planted in tropical or subtropical regions. The cultivar Shuhui498 (R498) is a major restorer line in a three-line hybrid system planted in Sichuan Province, located in southwest China. We developed a segregating population from a cross between R498 and Nipponbare and planted the population to $\mathrm{F}_{3}$ generation. The tissues collected for RNA-seq include: roots ( 4 weeks), stems (6 weeks), leaves ( 2 and 6 weeks), spikelets (2-3 days before and 2-3 days after heading) and hulls (7 and 14 days after heading).

For WGS sequencing, high-molecular-weight genomic DNA was extracted from 10-day-old leaves of R498 (multiple seeds) with chloroform-isoamyl alcohol (24:1) followed by cetyltrimethylammonium bromide ${ }^{38}$ extraction and precipitation using 0.7 volumes of isopropanol. Then they were washed by $1 \mathrm{ml} 70 \%$ ethanol and dissolved in sterile deionized water. RNA from the rice tissues were extracted with TRIzol (Thermo Fisher Scientific, Waltham, MA, USA). Following the cetyltrimethylammonium bromide method, the DNA of the $\mathrm{F}_{3}$ population was extracted from the green leaves of individuals planted in the field.

WGS SMRT sequencing on PacBio platform and de novo assembly. The sequencing libraries were prepared with the standard protocol from PacBio and sequenced by Wuhan Nexomics (http: //www.nextomics.cn) on the PacBio long read sequencing instrument, model RSII, with both P5 Polymerase Binding and C3 Chemistry Kits and P6 Polymerase Binding and C4 Chemistry Kits.

The raw PacBio reads were error-corrected and assembled using the $\mathrm{PBcR}^{27}$ pipeline with default low stringent parameters: -length 1000 -partitions 300 -sensitive -threads 8 -noclean merSize $=14$ asmOvlErrorRate $=0.10$ asmUtgErrorRate $=0.07$ asmCgwErrorRate $=0.10$ asmCnsErrorRate $=0.10$ utgGraphErrorRate $=0.07$ merylMemory $=32000$. The high stringent criteria for CA used in the assembly of the whole genome was AS_OVL_ERROR_RATE $=0.04$ AS_OVERLAP_ MIN_LEN $=1000$. The corrected sequences were $17 \mathrm{~Gb}$, and the longest $10 \mathrm{~Gb}$ sequence was selected automatically by $\mathrm{PBcR}$ for assembly. The Falcon pipeline (v0.3.0) (https://github.com/PacificBiosciences/FALCON-integrate) was used to do an independent whole genome assembly using the following parameters:

length_cutoff_pr $=8000$ pa_HPCdaligner_option $=-\mathrm{v}-$ dal128 - t16 -e.70 -11000 s1000 ovlp_HPCdaligner_option $=-\mathrm{v}-$ dal128 -t32 -h60 -e.96 -1500 -s1000 pa_DBsplit_option $=-x 500-s 400$ ovlp_DBsplit_option $=-x 500-s 400$ falcon_sense option =--output_multi --min_idt 0.70 --min_cov 4 --local_match_count_threshold

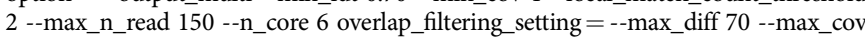
300 --min_cov 2 --bestn 15. The CANU (http://canu.readthedocs.org/) pipeline was run with the default parameters. The contaminated sequence contigs from bacteria, fungi or human genome were removed after BWA aligning to the downloaded sequences from GenBank with alignment length $>1 \mathrm{~kb}$.

WGS sequencing on Illumina platform and de novo assembly. The sequencing libraries with insert size that peaked at $450 \mathrm{bp}$ were prepared using a PCR-free protocol (Illumina Kit FC-121-3001; Illumina Inc., San Diego, CA, USA). After library profile analysis was performed by an Agilent 2100 Bioanalyser (Agilent Technologies, Santa Clara, CA, USA) and quantitative PCR quantification, the library was sequenced to $2 \times 150 \mathrm{bp}$ on the Illumina HiSeq X 10 platform (Illumina).

The short reads were filtered by removing adaptor contaminations and lowquality reads with base N's or more than $10 \%$ of bases with a quality score $<20$. The de novo assembly of the WGS short reads was performed by SOAPdenovo (http://soap.genomics.org.cn/SOAPdenovo.html), with $\mathrm{kmer}=83$. The assembled sequences were filtered by removing those aligned to microbial and human genomes (minimum aligned length is $100 \mathrm{bp}$ ) downloaded from GenBank with BWA-mem (http://bio-bwa.sourceforge.net/) default parameters.

RNA sequencing on Illumina platform and data analysis. RNA-seq libraries were prepared with Illumina TruSeq RNA Library Prep Kits v2, with insert size of $300-400 \mathrm{bp}$ and sequenced to $2 \times 144 \mathrm{bp}$ on the Illumina HiSeq 2500 platform (Illumina).

RNA-seq short reads were aligned to genome with Hisat2-2.0.1-beta (https://ccb.jhu.edu/software/hisat2/index.shtml), and the expression level of each gene (fragments per kilobase of exon per million fragments mapped) was computed by cufflinks v2.1.1 -u (https://github.com/cole-trapnell-lab/cufflinks). A gene is considered to be expressed if its fragments per kilobase of exon per million fragments mapped $>0$.

For RNA-seq-based genome coverage computation and SNP calls, only uniquely mapped reads were selected, and SNPs were obtained by GenomeAnalysisTK-2.7-2, UnifiedGenotyper, with filtering criteria of depth $\geq 5$, SNP quality $\geq 50$, cluster SNP within 10 base.

Genome sequence alignments and SNP calling. We used the BWA-aln and BWA-mem (http://bio-bwa.sourceforge.net/) default setting to align all Illumina short reads to the assembled genome. After alignment, we used SAMtools ${ }^{39}$ to filter out low-quality (mapping quality <30) alignments, and then used the Genome Analysis Toolkit (GATK) (https://www.broadinstitute.org/gatk/) UnifiedGenotypers to call SNPs. The SNPs were filtered using the GATK VariantFiltration program with the following criteria: clusterWindowSize:10, MQ0 $>=4 \& \&((\mathrm{MQ} 0 /(1.0 * \mathrm{DP}))>0.1)$, $\mathrm{QUAL}<50.0, \mathrm{DP}<5$. The PacBio raw reads were aligned to the genome by $\mathrm{BLASR}^{7}$ or BWA.

Genetic population sequencing and map construction. An $\mathrm{F}_{3}$ population of 365 individuals from a cross of R498 and Nip were selected to perform double restriction enzyme (BamHI and $M s p I)$ GBS sequencing ${ }^{26}$. DNA samples ( $\sim 300 \mathrm{ng}$ per sample) were digested for $2 \mathrm{~h}$ at $37^{\circ} \mathrm{C}$ with $\mathrm{MspI}$ and BamHI (New England BioLabs Inc., Ipswitch, MA, USA) in $15 \mu \mathrm{l}$ volumes and heated for $20 \mathrm{~min}$ at $65^{\circ} \mathrm{C} . \mathrm{MspI}$ and BamHI adapters were ligated to sticky ends by adding $5 \mu \mathrm{l}$ of ligation mix containing T4 DNA ligase (NEB) at $22^{\circ} \mathrm{C}$ for $2 \mathrm{~h}$ and incubated at $65^{\circ} \mathrm{C}$ for $20 \mathrm{~min}$ to inactive the T4 ligase. Sets of 96 digested DNA samples, each with different barcode adapters were combined ( $5 \mu \mathrm{l}$ each) and purified using a commercial kit (QIAquick PCR Purification Kit; Qiagen Inc., Valencia, CA, USA) according to the manufacturer's instructions. DNA samples were eluted in a final volume of $45 \mu \mathrm{l}$ and those ranged from 300-400 bp were size selected on $2 \%$ agarose gel. Amplification of the library was performed using 11 PCR cycles containing an Illumina common adapter primer and an index adapter primer. Sequencing was conducted by using an Illumina HiSeq 2500 instrument (Illumina) to generate $2 \times 125$ bp reads.

The short reads were aligned to R498 PBcR LS contigs, and SNPs were called as described above. After filtering, we identified a total of 2,087,353 homozygous SNPs between the parents on 1,158 contigs. These SNPs were used to filter out unmatched SNPs or extremely unevenly distributed SNPs $(<5 \%$ for any one parent genotype) in the $\mathrm{F}_{3}$ population. Finally, we obtained 491,128 SNPs on 787 contigs for linkage map construction. Sliding windows of size 50 SNPs were used to create SNP bins to find recombination sites. JoinMap4.1 (https:// www.kyazma.nl/index.php/mc.JoinMap/) ML methods were used to cluster the bins into LGs, and then the MstMap (http://alumni.cs.ucr.edu/ yonghui/ mstmap.html) Kosambi model was used to compute the order of the bins.

Fosmid library sequencing and assembly. The R498 fosmid library was constructed by following the protocol of the CopyControl Fosmid Library Kit (Epicentre Biotechnologies, Madison, WI, USA) using high-quality DNA $\left(>200 \mathrm{ng}^{-1} \mathrm{l}^{-1}\right.$ ) extracted with a DNeasy Plant Mini Kit (Qiagen) from 2-week-old leaves of R498. The library consisted of 1-2 million independent clones which were stored in four pieces of 384-well plates of 1,000-1,500 clones per sample. The average clone insert size was $36 \mathrm{~kb}$ and the whole library was equivalent to about 100-200 $\times$ genome coverage. We selected 564 pooled samples for sequencing in six barcoded GBS sequencing libraries, each containing 96 pools.

The fosmid clones were grown in LB broth with $12.5 \mu \mathrm{g} \mathrm{ml}^{-1}$ chloramphenicol in 2-ml-deep 96-well plates shaking at 400 r.p.m. under $37^{\circ} \mathrm{C}$ for $10 \mathrm{~h}$ and continued for $6 \mathrm{~h}$ after adding $0.1 \mathrm{mg} \mathrm{ml}^{-1} \mathrm{~L}-(+)$-arabinose for inducing multiple copies. The plates were centrifuged for $50 \mathrm{~min}$ at 4,000 r.p.m. to collect bacteria, and the fosmid DNA was extracted according to a standard user manual of the plasmid DNA purification NucleoSpin 96 Flash Kit (Macherey-Nagel, Düren, Germany). DNA was finally dissolved in $40 \mu \mathrm{l}$ of sterile deionized water. We used the same GBS protocol ${ }^{26}$ as used for $F_{3}$ population sequencing to sequence the fosmid DNA library. The libraries were sequenced to $2 \times 125$ bp on the Illumina HiSeq 2500 platform (Illumina) (a total of $6.3 \mathrm{~Gb}$ sequences, $3 \times$ on each tag).

GBS sequence tags in each pool were aligned to the corrected PacBio reads by BWA-mem, and PacBio reads were selected according to the following criteria: up to $20 \times$ with the highest coverage $\times$ identity by sequence tags, with alignment identity at least $97 \%$. Only aligned GBS tags in full length were used to compute sequence coverage of PacBIo reads except at the ends of the PacBio reads, where 
$>50 \%$ of the GBS tags must be aligned. We assembled these long reads using Falcon with the following parameters: ovlp_HPCdaligner_option $=-\mathrm{v}-\mathrm{dal} 4-\mathrm{t} 32$ h60 -e.90 -1500 -s1000, overlap_filtering_setting = --max_diff 50 --max_cov 50 -min_cov 1 -bestn 10. All contigs were put together after assembly, and contigs shorter than $10 \mathrm{~kb}$ were removed. The fosmid contigs were further assembled into a non-redundant genome with CANU.

Construction of super-contigs. An overlap graph was constructed using WGS contigs as nodes and fosmid contigs as edges. A WGS contig as node has one or two ends connecting to overlapping fosmid contigs. A fosmid contig as edge has two sequence overlaps on its opposite ends. The score of an edge was defined as:

edge_score $=$ left_overlap_length $*$ left_identity + right_overlap_length $*$ right_identity

The weighted score for a pair of nodes is the sum of scores of all the edges between them satisfying the predefined thresholds on the overlap identity and length, and the overhang length. The weighted scores for all pairs of connected nodes were recomputed during each step of the connection process using the valid edges. The length of an edge is defined as the sequence length on the fosmid contig between the two overlaps connecting two nodes:

edge_length = right_overlap_start_position - left_overlap_end_position

Note that an edge length can be negative if a pair of WGS contigs has sequence overlap between them (Fig. 1e).

The WGS contigs were connected with fosmid contigs to form super-contigs as follows. First overlap graphs were built by aligning all WGS contigs against themselves and aligning all fosmid contigs to all WGS contigs with BWA to find sequence overlaps. This step was repeated for all newly constructed super-contigs as necessary (as sometimes BWA is unable to find all valid overlaps between contigs, the query and target were exchanged and BWA alignment was redone).

Second, nodes were iteratively merged to represent neighbouring contig pairs in a LG with the best-weighted score of all edges with overlap alignment identity $>99 \%$, matched length $>5 \mathrm{~kb}$ and overhang of $<1 \mathrm{~kb}$. They were not merged if there were conflicting overlaps (that is, one end of a contig is connected to more than one WGS contigs with the same weighted score). After each node merge, any edges connecting to the already merged contig ends were removed to reduce conflicts for other nodes.

Thirdly, nodes were iteratively merged to represent neighbouring contig pairs using the same criteria as described above, except the identity was reduced to $98 \%$.

Fourth, the previous two steps were repeated while also allowing connections between an anchored contig and an unanchored WGS contig. Each WGS contig was used only once and the shortest edge was selected in case of conflicts. Once an unanchored contig was merged to an anchored one, its connections to the contigs on other LGs were all removed. This step was repeated until no more reliable connections were left and then repeated again with alignment identity further reduced to $97 \%$. This step allowed the extension of the anchored contigs by connecting to the unanchored WGS contigs after all possible neighbouring pairs were connected.

Fifth, chimeric contigs resulting from the original WGS assembly were found and fixed. If one contig or super-contig had sequence overlap to the middle of another contig with overlap length $>5 \mathrm{~kb}$, identity $>98 \%$ and overhang $>1 \mathrm{~kb}$, and the overlap was supported by fosmid contigs, then the latter was split and they were connected. The cleaved sequences of $>30 \mathrm{~kb}$ were used to fill gaps elsewhere.

Sixth, misassembled WGS contigs around gaps were replaced with more reliable WGS contigs and whole connection process was repeated. Unfilled gaps (without reliable overlaps to any WGS or fosmid contigs) outside of the centromere regions after the previous step were likely due to the misassembled WGS contigs around the gaps. These misassembled contigs were replaced with more reliable contigs (for example, those assembled under more stringent criteria) that were matched to the flanking regions for further connection.

Finally, the unanchored WGS contigs not incorporated onto the chromosomes were connected with fosmid contigs wherever possible.

To output the sequences of the super-contigs, the sequences from the WGS contigs were used for the overlapping regions except for edges with negative length where the sequences from the fosmid contigs were used for the overlapping regions due to the low sequence quality in the overlapping regions of many WGS contig pairs.

BioNano data generation and analysis. R498 leaves from 10-day-old seedlings growing in dark conditions were collected. High-molecular-weight DNA extraction and DNA labelling (with Nt.BspQI nicking endonuclease) were both performed according the standard protocols provided by BioNano Genomics. Labelled DNA samples were loaded and run on the Irys system (BioNano Genomics) (service provided by BGI http://www.bgitechsolutions.com/index.html). Singlemolecule maps of $>100 \mathrm{~kb}$ were collected.

The single-molecule maps were corrected by the IrysView (v2.5.1) software (BioNano Genomics) with parameter '-autonoise', which used the PBcR LS contigs to obtain noise parameters for molecule mapping and assembly. The de novo assembly, hybrid assembly and detection of SVs were all done using the same Irys assembler and aligner, with auto selected parameters, and a minimum length of $150 \mathrm{~kb}$. The SVs were stored in a file named ${ }^{*}$.InDel that was output from IrysView compare module. For hybrid assembly with module HybridScaffold, the sequence contigs were split wherever conflicts were found between them and the genome maps.

Error correction and assembly of two organelle DNAs. To correct assembly errors in the indels $>10 \mathrm{~kb}$ identified with genome maps, two WGS contigs of $>100 \mathrm{~kb}$ around each indel were selected as starting and ending nodes on the overlap graph to enumerate the best-scored paths between them (Supplementary Fig. 5). In case that more than one valid aligned region was found between a WGS contig and a fosmid contig, a link was added to the overlap graph for each aligned region. The path matching to the genome map with the same length and nicking sites was selected as the replacing path. For local reassembly, the corrected PacBio sequences were aligned by BWA-mem to the misassembled regions with indels $<10 \mathrm{~kb}$ (extending $30 \mathrm{~kb}$ to each side of the indels) in the genome or the misassembled cpDNA and mtDNA super-contigs. The sequences with alignment identity $>98 \%$ and matching length $>3 \mathrm{~kb}$ were selected for CANU reassembly with default parameters. The reconnected or reassembled sequences were used to replace the corresponding erroneous regions in the pseudomolecules.

Two small super-contigs of 593 and $619 \mathrm{~kb}$ after connection were found to be homologous to Nip cpDNA and mtDNA, respectively. After reassembly, the largest cpDNA contig contained a circular DNA of $134 \mathrm{~kb}$. The reassembled mtDNA contigs were connected by fosmid contigs with alignment identity $>99 \%$ and overlap length $>10 \mathrm{~kb}$ into a contig of $414 \mathrm{~kb}$. The contig ends were extended with the corrected PacBio reads and reassembled for several rounds, and again were connected with the fosmid contigs until a circular DNA of $527 \mathrm{~kb}$ was obtained. The raw SMRT reads were aligned back by BLASR to mtDNA to assure the complete coverage and lack of breaking points as shown in Supplementary Fig. 8.

Centromere and telomere sequence identification. Centromere-specific (the RCS2 family ${ }^{40}$ ) repeats were aligned by BWA-mem (default parameters) to the final R498 genome and the assembled contigs to identify centromere-related sequences. The centromere repeat regions in R498 were defined as the regions that contained all full units of the RCS2 family. The telomere sequence $5^{\prime}$-AAACCCT- $3^{\prime}$ and all different orders of the seven bases were searched directly.

Postassembly examination of WGS contigs. All initially assembled WGS contigs were aligned to R498 genome using BWA-mem. Alignment identity $\geq 97 \%$ and coverage $\geq 95 \%$ were used to identify contigs that were incorporated into the final genome assembly. Contaminated microbial DNA was identified with BWA alignment coverage $\geq 95 \%$. R498 cpDNA and mtDNA were used to identify chloroplast and mitochondrial contigs with BWA alignment identity $\geq 95 \%$ and coverage $\geq 95 \%$. Centromere-related contigs were identified with BWA alignment coverage $\geq 95 \%$. The rest of the contigs were designated as unclassified. For computing genome coverage, one contig was allowed to match to only one bestmatched genome location. An erroneous contig was defined if it was aligned to one block with overhang $>1 \mathrm{~kb}$ ( $>100 \mathrm{bp}$ for short read assembly) or aligned to two or more non-overlapping blocks. For short read assembly, the contigs were filtered by aligning them to microbial and human DNAs with BWA-mem and then mapped to the R498 assembly including cpDNA and mtDNA with BLAT default parameters. The unmapped contigs were aligned to themselves with BLAT to remove those redundant sequences that were covered on $>90 \%$ of their length by a longer sequence.

Gene and repeat annotations. The Nip and R498 genes were first predicted by an evidence-based pipelin $e^{29}$, using rice and monocot cDNAs/ESTs downloaded from GenBank, proteins downloaded from SwissProt, and rice RNA-seq data that were downloaded from GenBank short read archive (http://www.ncbi.nlm.nih.gov/Traces/ sra/sra.cgi) and assembled by SOAPdenovo-trans (http://soap.genomics.org.cn/ SOAPdenovo-Trans.html) as evidence. We annotated motifs and domains using InterProScan (http://www.ebi.ac.uk/Tools/pfa/iprscan5/). Gene functions were further annotated according to the best-matched proteins in Brachypodium distachyon (http://genome.jgi.doe.gov) or maize (ftp://ftp.ensemblgenomes.org) databases using blastp with $30 \%$ minimal identity and coverage. To make reliable comparison of the genes between the two genomes, we created a core set of proteincoding genes by removing low-confidence genes as follows: (1) sharing homology to transposons in plants by more than 100 amino acids or $50 \%$ in DNA length, (2) EST only single-exon genes with protein length $<50$ amino acids or covering $<70 \%$ of another homologous genes in the former's full protein length (as pseudogenes). The coordinates of the genes in MSU7 (38,869 valid genes) and RAP (35,472 valid genes) were compared to our annotated Nip core gene set to find overlapping genes among them. The comparison of genes between R498 and Nip or within R498 was conducted by protein BLAST (ncbi-blast-2.2.28 + -evalue 1e-5) using the longest protein in each gene. The sequence identity and coverage were computed based on the aligned length and total length of each gene.

The repetitive sequences in both R498 and Nip genomes were annotated by combining $a b$ initio and homology-based methods. First, an $a b$ initio repeat library was predicted for each genome with LTR_FINDER (http://tlife.fudan.edu.cn/ ltr_finder/); the type of each repeat family was assigned by homology to PGSB Repeat Element Database (http://pgsb.helmholtz-muenchen.de/plant/recat/). 
Second, this library was combined with Repbase (http://www.girinst.org/repbase) for identifying all homologous repeats throughout the genome by RepeatMasker (http://www.repeatmasker.org/) with WU-BLASTX as search engine. Third, since many of the identified repeats overlapped with each other, the overlapping repeats in the same class were combined together. Among the overlapping repeats belonging to different classes, the longer ones were split into pieces at the overlap boundaries and the redundant parts were discarded to ensure only one class assignment for each repeated sequence. Fourth, the tandem repeats were further identified with Tandem Repeats Finder (http://tandem.bu.edu/trf/trf.html). The solo-LTRs and intact LTRs were further classified with LTRharvest (https://omictools.com/ltrharvest-tool).

Genome comparison and SV identification. Dot plot alignment was conducted with MUMmer ${ }^{41}$ with -mincluster 500 . Synteny analysis was also conducted using SyMAP v4.2 (http://www.agcol.arizona.edu/software/symap). The genome coverage of short reads was computed by BedTools coverage (http://bedtools.readthedocs.org). We identify SNPs and indels $<500$ bp between R498 and Nip genomes using MUMmer with the following four parameters: (1) nucmer -maxmatch -c $90-140$, (2) delta-filter-1, (3) show-snps and (4) show-diff. In addition, we used the collinear, non-overlapping, best-matched blocks of minimum 200 bp generated from MUMmer to identify unaligned sequence gaps $\geq 100$ bp between R498 and Nip, and between each draft genome and one of the two reference genomes. The unaligned gap length between R498 and Nip was recomputed by removing the sequences of $\geq 200 \mathrm{bp}$ with homology to the other genome. The recomputed gap length was treated as the PAV length. For the unaligned gap sequences in a reference genome relative to the draft genomes, we aligned their whole-genome short reads to the reference genome and kept only those $\geq 500$ bp with sequence coverage of $<25 \%$ as PVs. Two PVs on a reference genome relative to two draft genomes were considered to be shared if they overlapped with each other by $>75 \%$ of their length.

The genes fully enclosed in the PVs between R498 and Nip were filtered by removing those with matching sequences of $\geq 200 \mathrm{bp}$ to the other genome; that is, only those genes without significant homology to the other genome were retained for further analysis. For GO enrichment analysis, we used the whole gene set in each genome as background to do Fisher test on the PV-enclosed genes.

Data availability. All analyses and quality control steps were coded in Perl scripts or Linux shell commands/scripts except where stated explicitly. The custom Perl codes for building overlap graphs and generating final sequences are provided as Supplementary Software. The sequence reads are available at The Genome Sequence Archive (GSA) (http://gsa.big.ac.cn/index.jsp) under project PRJCA000313. The genome assembly of R498, and the data from WGS sequencing, population sequencing and fosmid sequencing have been deposited under NCBI BioProjects PRJNA318714, PRJNA318714, PRJNA318826 and PRJNA340081. The accessions to R498 assembly are CP018157-CP018170, and the accessions to genome sequencing data are SRP073415 and SRP073521. The R498 genome assembly and the annotated genes for both R498 and Nipponbare are also accessible at http://www.mbkbase.org/R498.

\section{References}

1. Weischenfeldt, J., Symmons, O., Spitz, F. \& Korbel, J. O. Phenotypic impact of genomic structural variation: insights from and for human disease. Nat. Rev. Genet. 14, 125-138 (2013).

2. Zichner, T. et al. Impact of genomic structural variation in Drosophila melanogaster based on population-scale sequencing. Genome Res. 23, 568-579 (2013).

3. Marroni, F., Pinosio, S. \& Morgante, M. Structural variation and genome complexity: is dispensable really dispensable? Curr. Opin. Plant Biol. 18, 31-36 (2014).

4. Saxena, R. K., Edwards, D. \& Varshney, R. K. Structural variations in plant genomes. Brief. Funct. Genomics 13, 296-307 (2014).

5. Michael, T. P. \& VanBuren, R. Progress, challenges and the future of crop genomes. Curr. Opin. Plant Biol. 24, 71-81 (2015).

6. Ross, M. G. et al. Characterizing and measuring bias in sequence data. Genome Biol. 14, R51 (2013)

7. Chaisson, M. J. \& Tesler, G. Mapping single molecule sequencing reads using basic local alignment with successive refinement (BLASR): application and theory. BMC Bioinform. 13, 238 (2012).

8. Chin, C.-S. et al. Nonhybrid, finished microbial genome assemblies from longread SMRT sequencing data. Nat. Methods 10, 563-569 (2013).

9. Chaisson, M. J. P. et al. Resolving the complexity of the human genome using single-molecule sequencing. Nature 517, 608-611 (2014).

10. VanBuren, R. et al. Single-molecule sequencing of the desiccation-tolerant grass Oropetium thomaeum. Nature 527, 508-511 (2015).

11. Gordon, D. et al. Long-read sequence assembly of the gorilla genome. Science 352, aae0344 (2016)

12. Chaisson, M. J. P., Wilson, R. K. \& Eichler, E. E. Genetic variation and the de novo assembly of human genomes. Nat. Rev. Genet. 16, 627-640 (2015).

13. Lam, E. T. et al. Genome mapping on nanochannel arrays for structural variation analysis and sequence assembly. Nat. Biotechnol. 30, 771-776 (2012).
14. Pendleton, M. et al. Assembly and diploid architecture of an individual human genome via single-molecule technologies. Nat. Methods 12, 780-786 (2015)

15. Elert, E. Rice by the numbers: a good grain. Nature 514, S50-S51 (2014),

16. Ma, J. \& Bennetzen, J. L. Rapid recent growth and divergence of rice nuclear genomes. Proc. Natl Acad. Sci. USA 101, 12404-12410 (2004).

17. Li, Z., Pinson, S., Paterson, A. H., Park, W. D. \& Stansel, J. W. Genetics of hybrid sterility and hybrid breakdown in an intersubspecific rice Oryza sativa $\mathrm{L}$. population. Genetics 145, 1139-1148 (1997).

18. Ouyang, Y., Liu, Y.-G. \& Zhang, Q. Hybrid sterility in plant: stories from rice. Curr. Opin. Plant Biol. 13, 186-192 (2010).

19. International Rice Genome Sequencing Project. The map-based sequence of the rice genome. Nature 436, 793-800 (2005).

20. Garris, A. J., Tai, T. H., Coburn, J., Kresovich, S. \& McCouch, S. Genetic structure and diversity in Oryza sativa L. Genetics 169, 1631-1638 (2005).

21. Yu, J. et al. The genomes of Oryza sativa: a history of duplications. PLoS Biol. 3, e38 (2005).

22. Gao, Z.-Y. et al. Dissecting yield-associated loci in super hybrid rice by resequencing recombinant inbred lines and improving parental genome sequences. Proc. Natl Acad. Sci. USA 110, 14492-14497 (2013).

23. Schatz, M. C. et al. Whole genome de novo assemblies of three divergent strains of rice, Oryza sativa, document novel gene space of aus and indica. Genome Biol. 15, 506 (2014).

24. Zhang, J. et al. Extensive sequence divergence between the reference genomes of two elite indica rice varieties Zhenshan 97 and Minghui 63. Proc. Natl Acad. Sci. USA 113, E5163-E5171 (2016).

25. Huang, X. et al. A map of rice genome variation reveals the origin of cultivated rice. Nature 490, 497-501 (2012).

26. Sonah, H. et al. An improved genotyping by sequencing (GBS) approach offering increased versatility and efficiency of SNP discovery and genotyping. PLoS ONE 8, e54603 (2013).

27. Berlin, K. et al. Assembling large genomes with single-molecule sequencing and locality-sensitive hashing. Nat. Biotechnol. 33, 623-630 (2015).

28. Schmutz, J. et al. Quality assessment of the human genome sequence. Nature 429, 365-368 (2004).

29. Liang, C., Mao, L., Ware, D. \& Stein, L. Evidence-based gene predictions in plant genomes. Genome Res. 19, 1912-1923 (2009).

30. Ma, J., Devos, K. M. \& Bennetzen, J. L. Analyses of LTR-retrotransposon structures reveal recent and rapid genomic DNA loss in rice. Genome Res. 14, 860-869 (2004).

31. Weng, J. et al. Isolation and initial characterization of GW5, a major QTL associated with rice grain width and weight. Cell Res. 18, 1199-1209 (2008).

32. Gamuyao, R. et al. The protein kinase Pstoll from traditional rice confers tolerance of phosphorus deficiency. Nature 488, 535-539 (2012).

33. Michael, T. P. Plant genome size variation: bloating and purging DNA. Brief. Funct. Genomics 13, 308-317 (2014).

34. Salse, J. et al. Identification and characterization of shared duplications between rice and wheat provide new insight into grass genome evolution. Plant Cell 20, 11-24 (2008)

35. Burton, J. N. et al. Chromosome-scale scaffolding of de novo genome assemblies based on chromatin interactions. Nat. Biotechnol. 31, 1119-1125 (2013).

36. Zheng, G. X. Y. et al. Haplotyping germline and cancer genomes with highthroughput linked-read sequencing. Nat. Biotechnol. 34, 303-311 (2016).

37. Schneeberger, K. Using next-generation sequencing to isolate mutant genes from forward genetic screens. Nat. Rev. Genet. 15, 662-676 (2014).

38. Clarke, J. D. Cetyltrimethyl ammonium bromide (CTAB) DNA miniprep for plant DNA isolation. Cold Spring Harb. Protoc. 2009, doi:10.1101/pdb.prot5179 (2009).

39. Li, H. et al. The sequence alignment/map format and SAMtools. Bioinformatics 25, 2078-2079 (2009).

40. Dong, F. et al. Rice (Oryza sativa) centromeric regions consist of complex DNA. Proc. Natl Acad. Sci. USA 95, 8135-8140 (1998).

41. Kurtz, S. et al. Versatile and open software for comparing large genomes. Genome Biol. 5, R12 (2004).

\section{Acknowledgements}

This work was supported by the Chinese Academy of Sciences 'Strategic Priority Research Program' fund (XDA08020302) to C.L., the Chinese Academy of Sciences 'Outstanding Technical Talents' program fund to C.L. and National Natural Science Foundation of China grant (91535102) to S.L.

\section{Author contributions}

C.L., S.L. and L.Z. planned the project. C.L. designed the experiments and analytical protocols and wrote the manuscript. S.L. and P.Q. provided all the Shuhui498 seeds and tissues for RNA extraction. H.D., Q.G., Y.C., Z.C., B.M., Y.L., X.Y. and C.L. performed the data analysis. Y.Y., Y.M., M.Q. performed the WGS, RNA and population sequencing, fosmid construction and sequencing experiments. X.Z., J.W., K.L. and P.Q. planted rice materials. X.Z. created the segregating population. 


\section{Additional information}

Supplementary Information accompanies this paper at http://www.nature.com/ naturecommunications

Competing interests: The authors declare no competing financial interests.

Reprints and permission information is available online at http://npg.nature.com/ reprintsandpermissions/

How to cite this article: $\mathrm{Du}, \mathrm{H}$. et al. Sequencing and de novo assembly of a near complete indica rice genome. Nat. Commun. 8, 15324 doi: 10.1038/ncomms15324 (2017)
Publisher's note: Springer Nature remains neutral with regard to jurisdictional claims in published maps and institutional affiliations.

(c) (i) This work is licensed under a Creative Commons Attribution 4.0 International License. The images or other third party material in this article are included in the article's Creative Commons license, unless indicated otherwise in the credit line; if the material is not included under the Creative Commons license, users will need to obtain permission from the license holder to reproduce the material. To view a copy of this license, visit http://creativecommons.org/licenses/by/4.0/

(C) The Author(s) 2017 